\title{
Laryngeal High-Speed Videoendoscopy: Sensitivity of Objective Parameters towards Recording Frame Rate
}

\author{
Anne Schützenberger, ${ }^{1}$ Melda Kunduk, ${ }^{2}$ Michael Döllinger, ${ }^{1}$ Christoph Alexiou, ${ }^{3}$ \\ Denis Dubrovskiy, ${ }^{1}$ Marion Semmler, ${ }^{1}$ Anja Seger, ${ }^{1}$ and Christopher Bohr ${ }^{1}$

\begin{abstract}
${ }^{1}$ Division for Phoniatrics and Pediatric Audiology, Department of Otorhinolaryngology, Head and Neck Surgery, Medical School, FAU-Erlangen-Nürnberg, Bohlenplatz 21, 91054 Erlangen, Germany

${ }^{2}$ Department of Communication Sciences and Disorders, Louisiana State University, 64 Hatcher Hall, Baton Rouge, LA 70803, USA

${ }^{3}$ Section of Experimental Oncology and Nanomedicine (SEON), Department of Otorhinolaryngology, Head and Neck Surgery,
\end{abstract} \\ Medical School, FAU-Erlangen-Nürnberg, Glückstrasse 10a, 91054 Erlangen, Germany
}

Correspondence should be addressed to Anne Schützenberger; anne.schuetzenberger@uk-erlangen.de

Received 6 July 2016; Accepted 10 October 2016

Academic Editor: Markus Hess

Copyright ( 2016 Anne Schützenberger et al. This is an open access article distributed under the Creative Commons Attribution License, which permits unrestricted use, distribution, and reproduction in any medium, provided the original work is properly cited.

\begin{abstract}
The current use of laryngeal high-speed videoendoscopy in clinic settings involves subjective visual assessment of vocal fold vibratory characteristics. However, objective quantification of vocal fold vibrations for evidence-based diagnosis and therapy is desired, and objective parameters assessing laryngeal dynamics have therefore been suggested. This study investigated the sensitivity of the objective parameters and their dependence on recording frame rate. A total of 300 endoscopic high-speed videos with recording frame rates between 1000 and $15000 \mathrm{fps}$ were analyzed for a vocally healthy female subject during sustained phonation. Twenty parameters, representing laryngeal dynamics, were computed. Four different parameter characteristics were found: parameters showing no change with increasing frame rate; parameters changing up to a certain frame rate, but then remaining constant; parameters remaining constant within a particular range of recording frame rates; and parameters changing with nearly every frame rate. The results suggest that (1) parameter values are influenced by recording frame rates and different parameters have varying sensitivities to recording frame rate; (2) normative values should be determined based on recording frame rates; and (3) the typically used recording frame rate of $4000 \mathrm{fps} \mathrm{seems} \mathrm{to} \mathrm{be} \mathrm{too} \mathrm{low} \mathrm{to} \mathrm{distinguish} \mathrm{accurately} \mathrm{certain} \mathrm{characteristics}$ of the human phonation process in detail.
\end{abstract}

\section{Introduction}

The human voice originates in the larynx from the oscillation of the two opposing vocal folds, Figure 2. Anatomical changes of the vocal folds and functional disorders can lead to changes in voice quality [1]. Endoscopic investigation of laryngeal structures allows direct visualization of vocal folds and their function during phonation. Currently, videostroboscopy [2] is the gold standard for investigating vocal fold function in clinical settings whereas high-speed videokymography and laryngeal high-speed videoendoscopy (HSV) are mainly used as supporting and additional imaging tools [3]. However, stroboscopy only allows the visualization of periodic vocal fold oscillations [3]. Stroboscopy is triggered by the vocal fundamental frequency and is therefore not applicable for smeared and not well-defined fundamental frequencies that are present in aperiodic vocal fold dynamics often associated with voice disorders [4]. In contrast, HSV permits the visual capture of the complete inter- and intracyclic behavior of the vocal fold dynamics [5-7]. Hence irregular and aperiodic vocal fold dynamics and also, for example, mucosal wave propagation can be visually observed and analyzed $[8,9]$. This is due to the temporal recording rate of HSV, typically 2,4 , and $8 \mathrm{kfps}$, being much higher than the fundamental vocal fold oscillation frequency of $100-300 \mathrm{~Hz}$ for normal human phonation [10, 11]. So far, HSV recordings have been mainly analyzed subjectively using perceptual evaluation of the data [11], which, depending on the study, seems to 
yield no advantages [12], minor to mild advantages [9, 13], or significant advantages [14] compared with stroboscopy. Consequently, these contradictory results do not justify the high acquisition costs of HSV. To establish HSV in daily clinical routine and actually to obtain advantages by applying HSV, image processing algorithms were developed to allow the computation of objective and quantitative measurements for HSV [15-17]. Based on these image processing algorithms, the first user-friendly software tools were suggested and applied in several studies $[6,18-20]$. The basis for a quantitative HSV analysis is the segmentation of the glottis [15], namely, the area between the two vocal folds, Figure 2. The segmentation of the glottis for all HSV frames yields the glottis area change over time and is called the glottal area waveform (GAW) [19], Figure 3(a). Using the GAW, objective and quantitative parameters can be computed describing the phonation process at the vocal fold level and providing information on periodicity and asymmetry characteristics of the vocal fold dynamics [21].

However, HSV is not yet established in clinical routine owing to several restrictive factors. Compared with stroboscopy, HSV hardware is rather expensive [22], there are no official guidelines for HSV footage analysis, and the additional benefit for daily clinical routine is still under discussion $[4,10,11]$. A further reason, despite existing studies with several hundred subjects [23], is the lack of normative parameter values and intervals, which are needed to determine the severity of pathological voice production. Also, studies have shown that there are significant parameter value differences between gender [23] and age groups [20, 24 ], complicating the definition of normative and clinically relevant data.

The HSV tool and its data can also influence the parameters of vocal fold vibratory function. These factors, which are expected to influence HSV parameters but have not been investigated thoroughly, include the recording frame rate (fps), the spatial resolution (pixels), and the analyzed interval length (i.e., the number of vocal fold oscillation cycles considered). Hence, in this study we investigated the influence of the HSV recording frame rate on quantitative GAW parameters, since many different recording rates have been used in existing studies. Popular HSV recording frame rates are $2 \mathrm{kfps}$ [7], $4 \mathrm{kfps}$ [6], and $8 \mathrm{kfps}$ [25]. Further, it was shown that too low HSV recording rates might also restrict subjective HSV analysis [9]. This study suggested, by analyzing healthy females, that $2 \mathrm{kfps}$ might be insufficient to judge subjectively certain vocal fold dynamic characteristics at oscillation frequencies above $200 \mathrm{~Hz}$. From the technical point of view, current high-end high-speed cameras enable frame rates of several $100 \mathrm{kfps}$ to be used. The latest studies in which these cameras were applied achieved recording frame rates of up to $20 \mathrm{kfps}$ and could record and analyze high soprano voices with frequencies up to $1568 \mathrm{~Hz}$ [26]. However, a disadvantage of very high recording frame rates is the potential decrease in spatial resolution. The camera model used in our work (Photron Fastcam SA1.1, Photron USA Inc., 2014), for example, permits a frame rate of $5.4 \mathrm{kfps}$ at a spatial resolution of $1024 \times 1024$ pixels. For $20 \mathrm{kfps}$, the spatial resolution is reduced to $512 \times 512$ pixels and for $75 \mathrm{kfps}$ to 512 $\times 128$ pixels.

In summary, HSV has the potential to support and supplant the clinical diagnosis of voice disorders and vocal fold vibratory dysfunction and objectively document therapy outcome. However, there is a need to establish normative data for HSV parameters to enhance the clinical importance and significance in clinical practice of this powerful imaging technique. In order to achieve this, one of the first aspects to establish is the influence of HSV recording frame rate on HSV parameters. To the best of our knowledge, there have been no studies on the investigation of the influence of the HSV recording frame rate on computed quantitative HSV parameters. However, as reported recently [20], there is a need to analyze the behavior and stability of HSV parameters with varying HSV recording rates. Hence, this aspect was the focus of the present study, in which we aimed to increase the awareness of this problem and to encourage a standardized computation of HSV parameters.

The goal of this study was not to acquire normative values based on many subjects but to demonstrate the sensitivity of objective HSV parameters to the recording frame rate. For this purpose, $300 \mathrm{HSV}$ recordings for one subject with varying frame rates were analyzed.

\section{Materials and Methods}

Ethical approval was obtained from the University of Erlangen-Nürnberg (number 290_13B). The participant gave informed, written consent prior to participation and this consent procedure was approved by the local committee. Experiments were performed in accordance with the Declaration of Helsinki (1964).

2.1. Data. HSV recordings were performed for a female subject during sustained phonation of the vowel /i/ at a comfortable pitch and loudness level for the participant. A $70^{\circ}$ endoscope was coupled to a gray-scale high-speed camera (Photron FASTCAM SA1.1 Model 675K-M1). HSV recordings were performed at $15 \mathrm{kfps}$ resolution in time at a spatial resolution of $768 \times 512$ pixels, being superior to current clinical HSV systems [20,21]. At the time of the experiment, the female participant was vocally healthy, a nonsmoker, and 45 years old. A total of $24 \mathrm{HSV}$ recordings were performed. The averaged phonatory fundamental frequency $F_{0}$ was $176 \pm$ $11 \mathrm{~Hz}$ with sound pressure levels around $74 \mathrm{~dB}$. Of the original 24 recordings, four were excluded owing to a lack of the desired number of glottis cycles, bad image quality, and full vocal fold length not being present. All HSV data analyzed had at least 106 oscillation cycles during sustained phonation to be segmented and analyzed. We chose to analyze 106 oscillation cycles since The National Center for Voice and Speech recommended (for acoustic signals) the analysis of a minimum sequence length of 100 oscillation cycles [27]; another study even proposed at least 110 cycles [28]. This yielded 20 original HSV recordings for further analysis.

2.2. Preprocessing: Glottis Segmentation. First, the frame rate of the $20 \mathrm{HSV}$ movies was reduced stepwise in $1 \mathrm{kfps}$ steps 
from 15 to $1 \mathrm{kfps}$. This yielded 15 movies, from 1 to $15 \mathrm{kfps}$, for each original HSV recording, resulting in a total of 300 HSV sample movies for further analysis. Next, the 15 movie samples from each original recording were image processed with identical segmentation parameters so as to eliminate influences from the image processing algorithms. For glottis segmentation, our in-house developed software Glottis Analysis Tools (GAT) was applied, providing the GAW over an interval of 106 oscillation cycles for each movie. From the GAW (Figure 3(a)), the objective and quantitative parameters were computed as described in the following.

2.3. HSV Parameters Computed from the GAW. A total of 20 parameters were computed and analyzed. The parameters considered were chosen because (1) they are already frequently applied in daily clinical routine and in other studies, (2) they reflect different characteristics of the vocal folds and glottis dynamics, and (3) they are computed on different spaces (spectral and time space of the GAW). Although there are certainly more objective parameters being used in HSV analysis $[8,23,29]$, the consideration of all possible HSV parameters would go far beyond one study. The primary goal of our study was to determine if there is a frame rate dependence among the HSV parameters. The 20 parameters investigated in this study are listed in Table 1 and they were used to examine (A) glottal dynamic characteristics, (B) glottal perturbation characteristics, and (C) glottal noise components.

2.4. Statistical Analysis. For statistical analysis, IBM SPSS Statistics Version 21 was used. All parameters were tested for normal distribution (Shapiro-Wilk test and Lilliefors modification of Kolmogorov-Smirnov test). The significance level was set to $5 \%(p<0.05)$. The tests showed that none of the parameters was normally distributed $(p<0.05)$. Therefore, nonparametric tests were used for further analysis.

The goal of the statistical analysis was to establish whether the parameter values change significantly with increase in recording rate from 1 to $15 \mathrm{kfps}$. For each parameter, it was checked if the 15 sample sets (recording rates $1-15 \mathrm{kfps}$ ) showed significant differences, indicating a dependence on the recording frame rate. Therefore, the Friedman test for comparing more than two dependent samples (here 15 samples) was applied. One sample consisted of the parameter values of all 20 movies for the corresponding recording rate (1-15 kfps). This means that 15 dependent samples (number of different recording rates) containing 20 values (number of HSV recordings) were compared. Except for two parameters (PA, OQ), the Friedman test showed that there are statistically significant differences for at least two samples (i.e., recording rates). However, it does not indicate which samples are different. Therefore, for further analysis, the Wilcoxon test for two dependent samples was applied. This means that, for each of the remaining 18 parameters, the sample with $1 \mathrm{kfps}$ was tested against each higher frame rate from 2 to $15 \mathrm{kfps}$, the sample with $2 \mathrm{kfps}$ was tested against the samples from 3 to $15 \mathrm{kfps}$, and so on. Hence, each parameter was tested 14 times for each frame rate. Therefore, a Bonferroni correction of the significance level $p$ was applied, yielding the new significance level $p=0.05 / 14=0.0036$.

\section{Results}

The behavior of the GAW parameters with respect to the HSV recording rate resulted in four main categories:

(i) Group 1: parameters remain stable; that is, the parameter values over the 15 frame rate samples are equal and hence independent of the recording rate.

(ii) Group 2: parameters remain unstable only until a certain frame rate is reached, and after that frame rate the parameter values are rate stable up to $15 \mathrm{kfps}$.

(iii) Group 3: parameters are stable within a certain HSV recording range interval (at least four recording rates) and change at lower and higher recording rates.

(iv) Group 4: parameters are highly dependent on HSV recording rate. These parameters change their values between each recording rate and are only stable for not more than three different HSV recording rates.

An overview of the computed mean values of the parameters for the corresponding recording rates is given in Figure 1. Recording rates showing no statistical differences are merged and the minimal and maximal values of corresponding means are given. Gray-intervals (JT, mJT, and HNR) indicate that these intervals do not show statistically significant differences from their left and right neighboring intervals. However, the intervals on the left and right borders of the gray-shaded interval are statistically different. Owing to space limitations, we have not given $p$ values, as a total of $105 \times 20=2100 p$ values would have had to be reported.

(A) Glottal Dynamic Characteristics. AMQ and MADR are highly recording rate dependent (group 4). These two parameters are stable over at most two HSV recording rates. The five parameters ASQ, CQ, RQ, SI, and SQ exhibit no changes from $8 \mathrm{kfps}$ and belong to group 2. GGI does not change after $12 \mathrm{kfps}$ (group 2). The two parameters OQ and PA seem to be independent of the recording rate, since they do not change at all and hence belong to group 1 .

(B) Glottal Periodicity and Perturbation Characteristics. All four parameters reflecting amplitude perturbation are in group 3. Whereas $\mathrm{AP}, \mathrm{mSH}$, and $\mathrm{SH}$ were stable between recording rates of 8 and $14 \mathrm{kfps}$, AVI did not change between 5 and $14 \mathrm{kfps}$. In contrast, both oscillation time perturbation measures JT and $\mathrm{mJT}$ are found in group 2. These two parameters did not change any further from a recording rate of $10 \mathrm{kfps}$.

(C) Harmonics and Noise Components. These parameters, except HNR, are computed over the frequency space of the GAW and change for nearly every recording frame rate. Whereas HI, NNE, and SPF belong to group 4, HNR shows stability for $8 \mathrm{kfps}$ and higher (group 2).

Considering the stability of the parameters, the following can be observed. Two (10\%) parameters (OQ, PA) are 


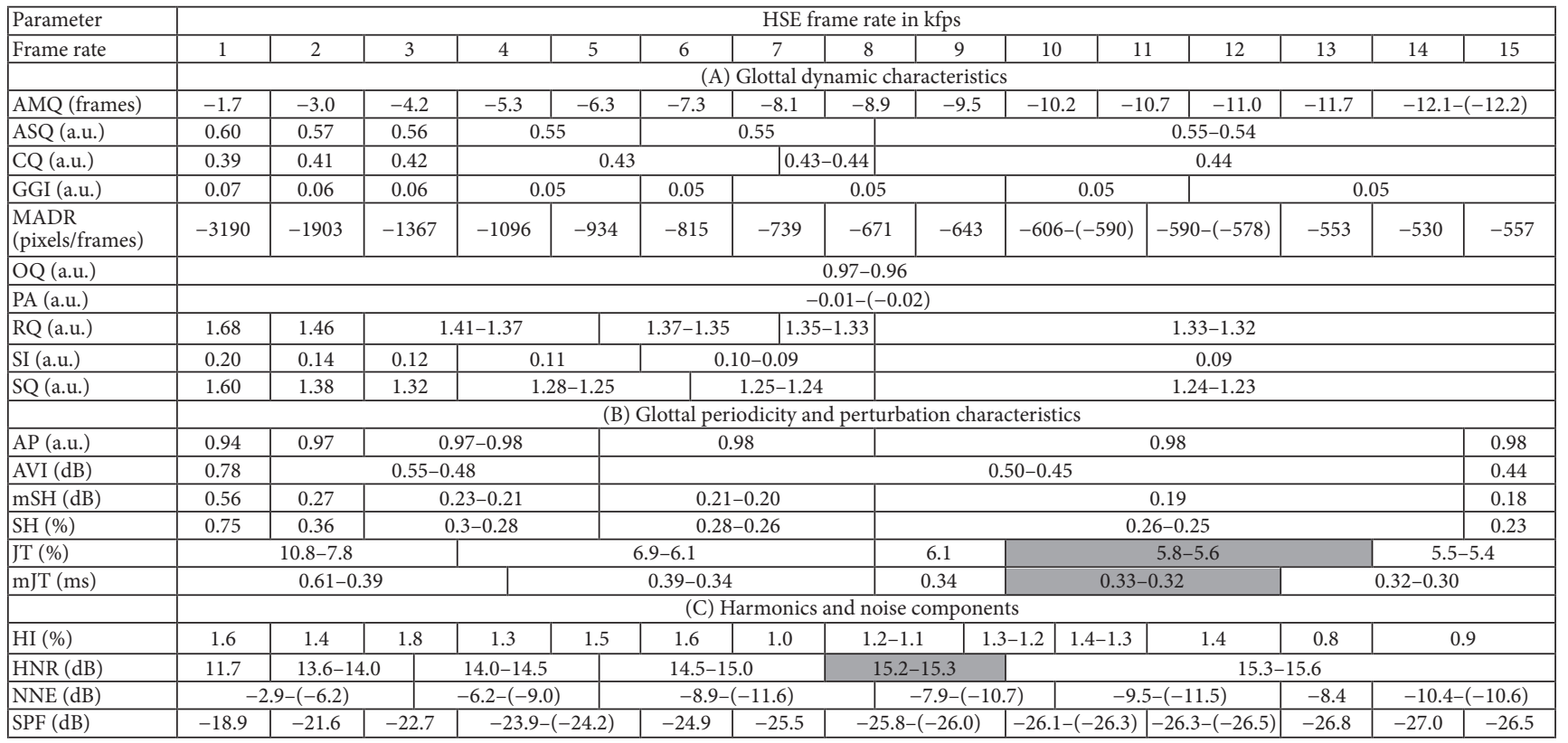

FIGURE 1: Computed mean parameter values for the different recording frame rates. For statistically not significantly different $p$ values $(p>$ $0.0036)$, the values are merged and the parameter value range is given. Gray-shaded areas mean that there is no statistical difference from the left and right recording frame rate intervals.

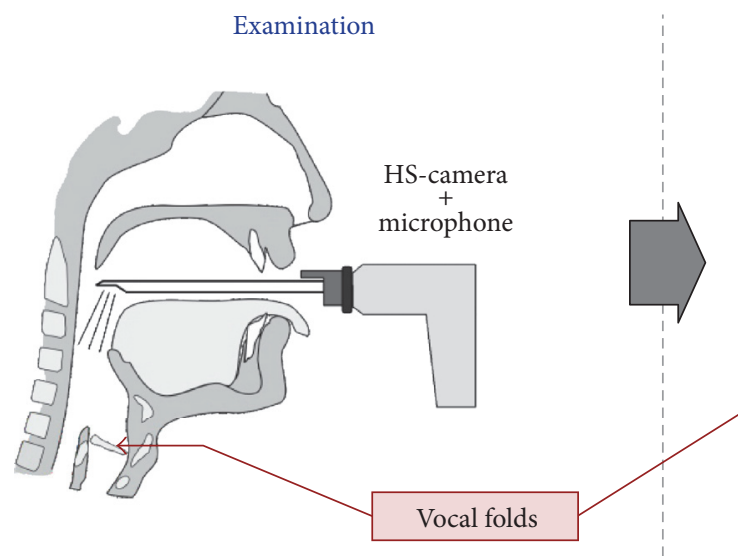

(a)

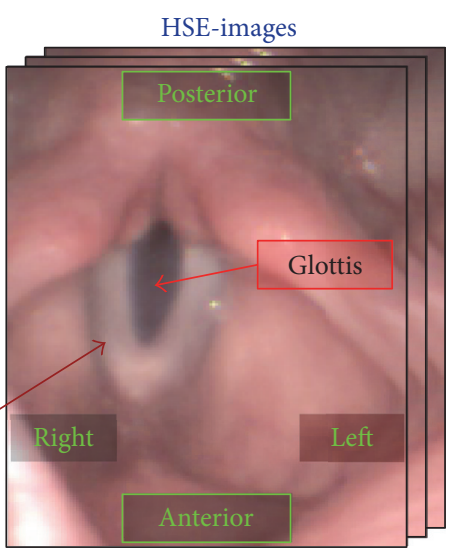

(b)

FIGURE 2: HSV recording setup and view of the vocal folds as seen through the camera. In (b), the dark glottis between the two vocal folds can be seen.

independent of the recording frequency (group 1). Nine parameters (45\%) (ASQ, CQ, GGI, RQ, SI, SQ, JT, mJT, and HNR) do not change any further after a certain recording rate (group 2), that is, after 8,10 , or $12 \mathrm{kfps}$. Four parameters (20\%) (AP, AVI, $\mathrm{mSH}$, and $\mathrm{SH}$ ) are stable over at least four frequency intervals (one parameter 5-14 kfps and three parameters 8$14 \mathrm{kfps}$ ) but change again at the highest recording rate (group 3). Five parameters (25\%) (AMQ, MADR, HI, NNE, and SPF) are highly dependent on the recording frequency and change for almost every recording rate (group 4). In particular, parameters computed in the frequency space (HI, NNE, and SPF) of the GAW seem to be highly dependent on the HSV recording rate.

To give a graphical impression of the behavior of the parameters with respect to the HSV recording rate, the averaged means and corresponding standard deviations for each recording rate are given in Figures 4-7. Additionally, the behavior of the parameters with respect to the HSV recording rates was subjectively rated by four experts. The experts were asked to define intervals for the parameters where they subjectively and visually assume the parameters to be stable and constant. All raters were in complete agreement for the chosen and indicated intervals as illustrated in Figures $4-7$. For 12 out of the 20 parameters, the experts confirmed the statistical results and rated identically. For five parameters (CQ, GGI, MADR, AVI, and $\mathrm{mSH}$ ), subjective evaluation of the stability by the raters showed that some of the statistically different intervals did not appear to change visually, whereas for three parameters (mJT, JT, and HNR) the experts visually evaluated changes where the statistics found no significant 


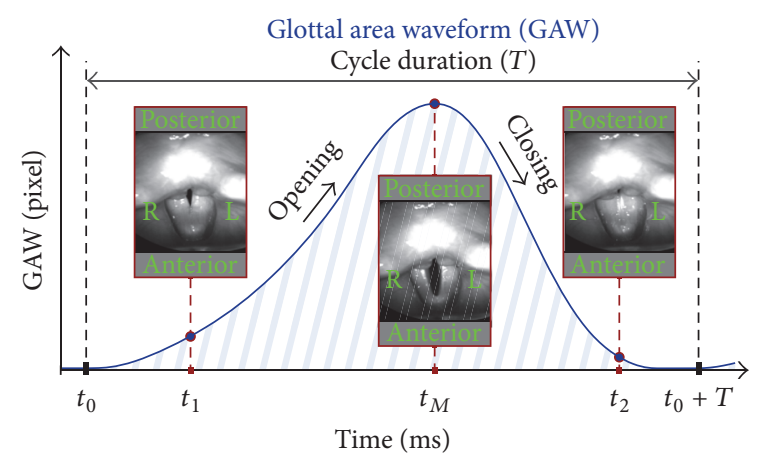

(a)

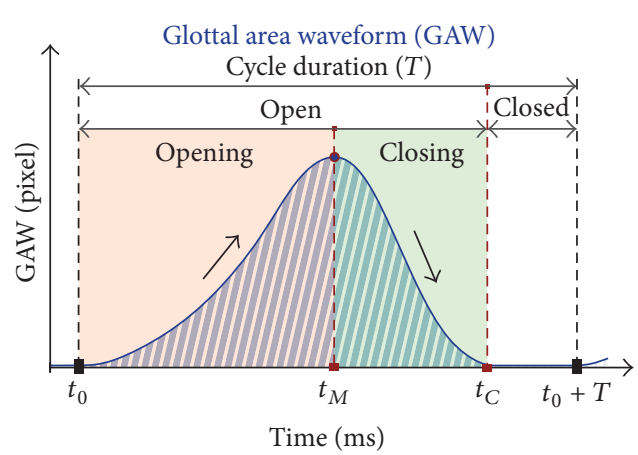

(b)

Figure 3: (a) Glottal area waveform (GAW) and the subdivision of the different oscillation states for computing the GAW parameters. (b) Definitions of GAW conditions used for parameter computation.

differences. However, these discrepancies were small and the subjective ratings closely reflected the statistical results as presented in Figure 1.

\section{Discussion}

The analysis on the dependence of HSV parameters on the HSV recording frame rate showed that $90 \%$ of the considered parameters change with frame rate. However, for the two parameters that did not show such a dependence this result will have to be confirmed in a larger set. The statistical results were mostly visually confirmed by the subjective perception of four experts.

In the following, the computed parameter values are discussed and compared with those obtained in previous investigations. If not explicitly noted, we refer to studies dealing with healthy female subjects investigated by HSV. When first mentioned that the configuration and details of each study are given in a table. The tables include means and standard deviations of the parameters, age and number of subjects, phonated tone, and fundamental frequency. The camera settings (recording frame rate and pixel resolution) and considered signal length are also given.

The purpose of comparing our parameter values with other study results was not to propose normative values but primarily to show that the variance of computed parameter values is often based on various applied HSV settings limiting the significance of computed absolute parameter values and also reducing the comparability between different studies. This emphasizes the need for detailed documentation within studies, but more important the need for well-defined HSV recording guidelines and normative parameter values based on potential influencing factors such as HSV recording frequency, spatial HSV resolution, age, and gender. This will then permit a better separation between healthy and disordered voice production and therefore enhance the clinical usefulness of HSV in the future.

(A) Glottal Dynamic Characteristics. The Amplitude Quotient (AMQ) belongs to group 4. In other words, AMQ values reach a stable state at 14 and $15 \mathrm{kfps}$. From 1 to $13 \mathrm{kfps}$ the samples are found to be statistically different. The values for
AMQ decrease continuously with increase in recording rates from -1.7 to -12.2 . The difference of AMQ at $4 \mathrm{kfps}(-5.3)$ compared with the value for $15 \mathrm{kfps}(-12.2)$ is high at $57 \%$. Compared with other studies (Table 2), our AMQ values at $4 \mathrm{kfps}$ are slightly lower. The results suggest that AMQ is highly influenced by the recording rate and that preferably very high recording rates should be used to achieve reliable results.

The Asymmetry Quotient (ASQ) belongs to group 2 and does not change after a recording rate of $8 \mathrm{kfps}(\mathrm{ASQ}=0.55$ $0.54)$. These values are similar to those of $0.59 \pm 0.05$ [41] and $0.52 \pm 0.06$ [21] obtained using $4 \mathrm{kfps}$. The changes in ASQ seem to be minor, with a maximum of 0.60 at $1 \mathrm{kfps}$, even though the differences are statistically significant. For higher recording rates, the ASQ values decrease continuously but to a small extent. For example, between 4 and $15 \mathrm{kfps}$ there is only a difference in the second decimal place. Hence, it appears that a $4 \mathrm{kfps}$ recording rate might be sufficient for ASQ computation.

For the Closing Quotient (CQ), the values do not change significantly from $8 \mathrm{kfps}$ (group 2). A previous study computed CQ at $0.45 \pm 0.07$ [21], which is very similar to our value at $15 \mathrm{kfps}(0.44)$, although they only used a recording rate of $4 \mathrm{kfps}$. Also, the presented values here at $4 \mathrm{kfps}$ are in the same range (0.43). Another study reported a lower CQ value of 0.34 [42]; there the parameter was called closing phase, Table 3.

Mehta et al. (2011) computed for seven healthy subjects (men and women) a lower CQ (0.26 \pm 0.08$)$ [43]. This CQ value is also below computed values for solely male groups presented previously: $0.40 \pm 0.09$ [46] and $0.38 \pm 0.11$ [41].

In summary, all CQ mean values in these studies are between 0.26 and 0.45 and are in the range reported by Holmberg et al. (1988) for healthy women [32]: 0.21-0.48. However, they computed their CQs from the glottal flow (i.e., inverse filtered) and not from the HSV-derived GAW.

The Glottis Gap Index (GGI) does not change after $12 \mathrm{kfps}$ and belongs to group 2. Overall, the values from GGI change only slightly from 0.07 to 0.05 , although showing statistically significant differences. However, the clinical significance of this finding needs to be further determined. The values 


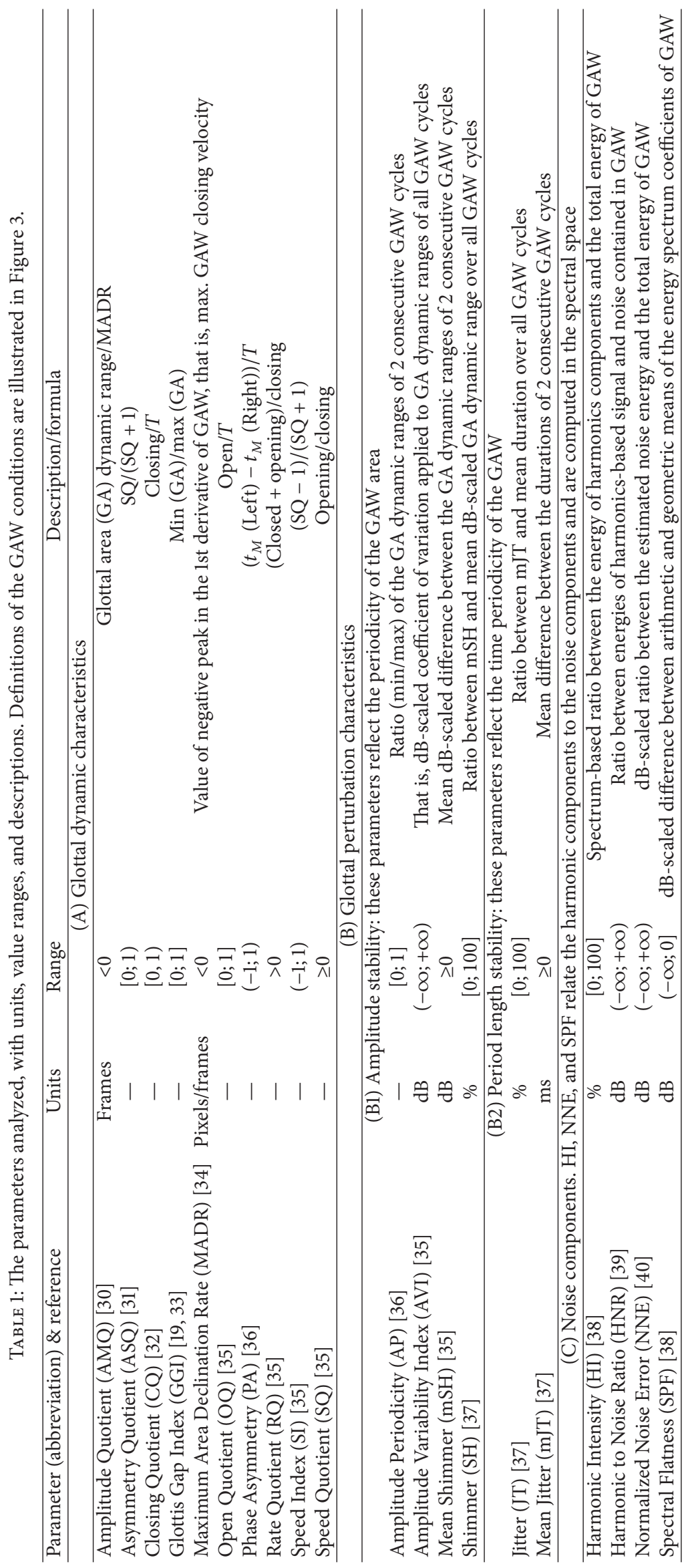



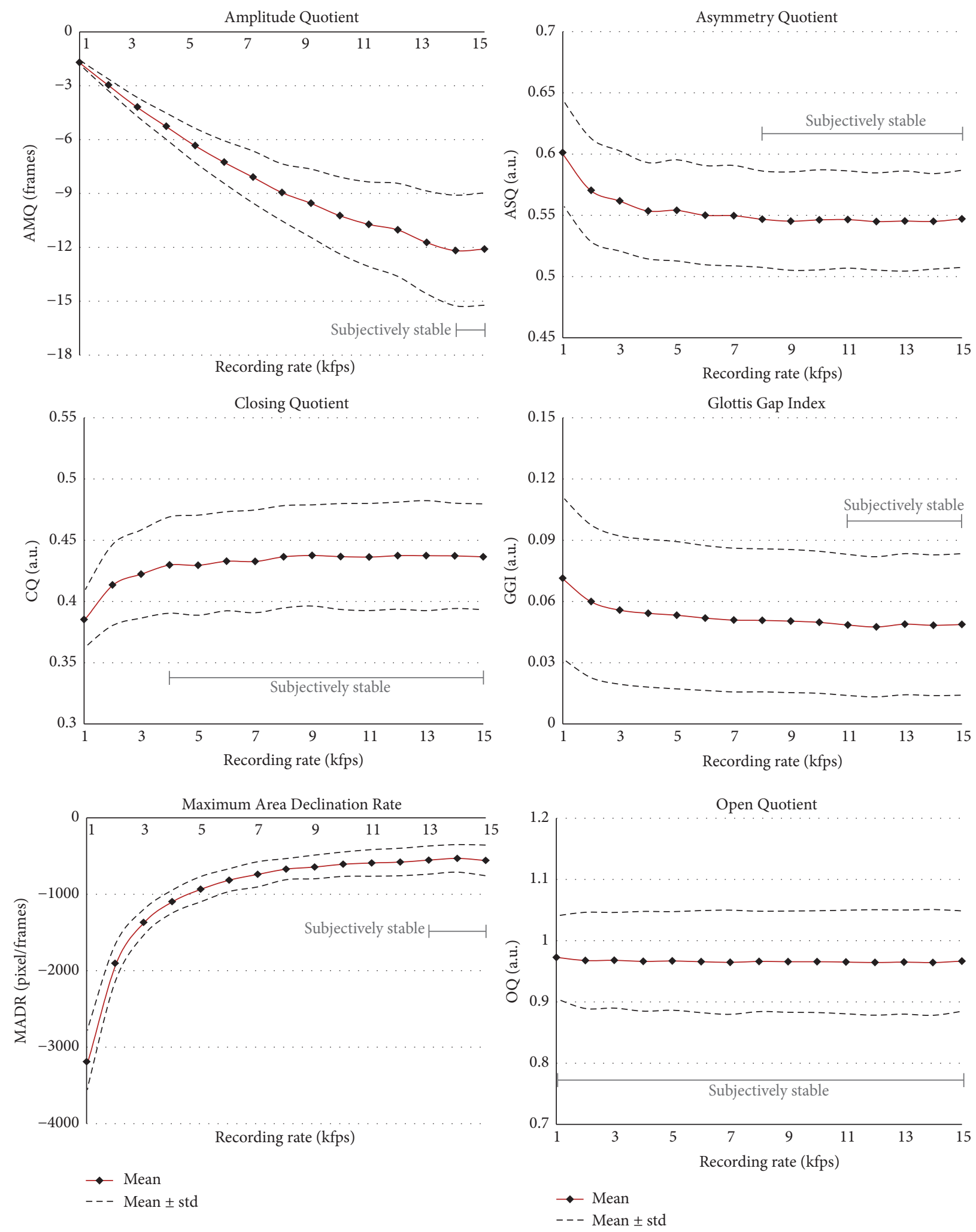

FIGURE 4: The first six parameters representing glottal dynamic characteristics. Means and standard deviations are given. The subjective rating of stable values over HSV recording frame rates is indicated. 

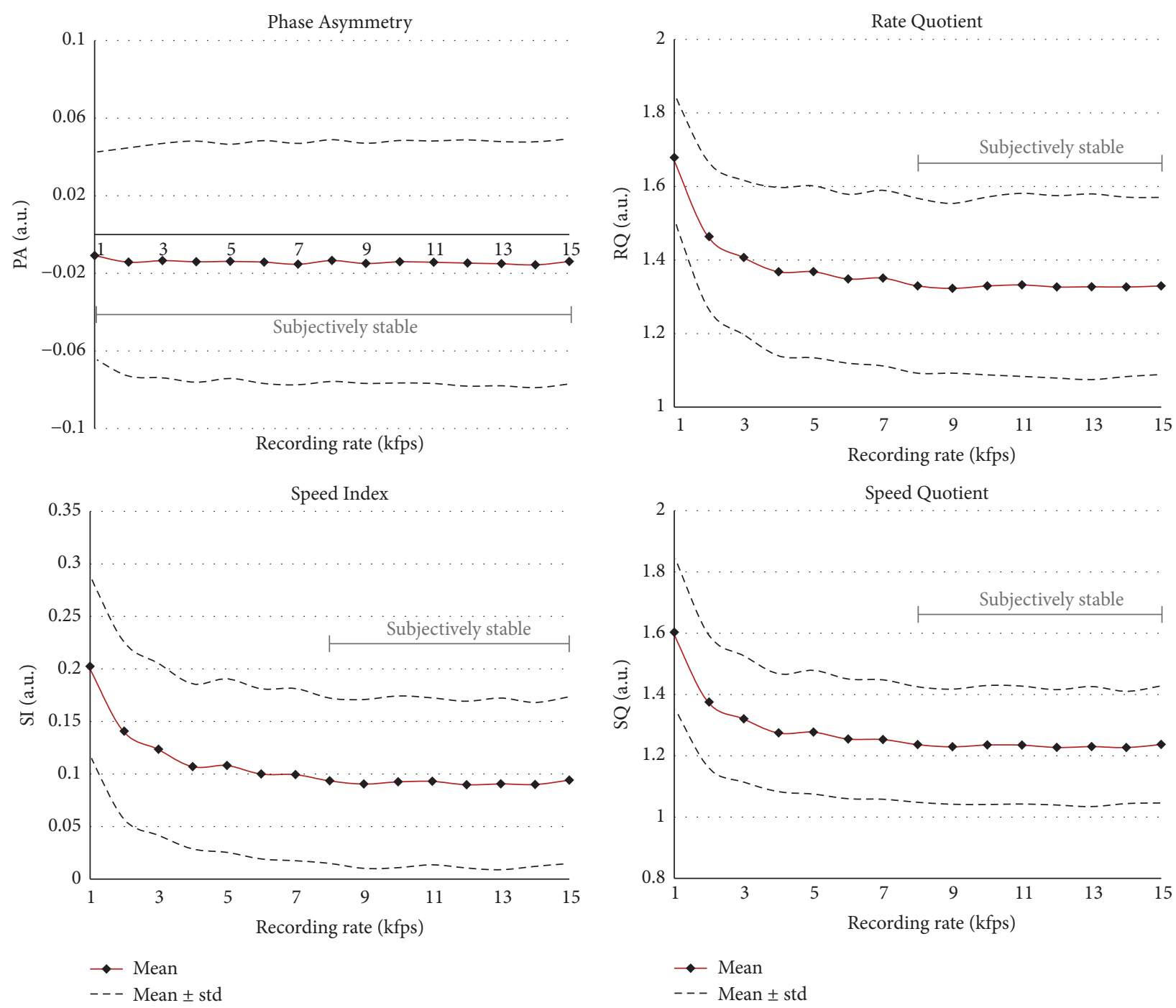

FIGURE 5: The other four parameters representing glottal dynamic characteristics. Means and standard deviations are given. The subjective rating of stable values over HSV recording frame rates is indicated.

confirm previous measurements for healthy female subjects [19]: $0.054 \pm 0.072$, Table 4 .

The Maximum Area Declination Rate (MADR) changes significantly for almost all recording rates (group 4). It increases continuously with increase in recording rate from -3190 ( $1 \mathrm{kfps})$ to -530 (14 kfps). This behavior is not surprising, since MADR was computed for pixels/frames and hence naturally decreases with increasing HSV recording rates. However, it seems that MADR stabilizes at $13 \mathrm{kfps}$ and above; that is, the values remain in the range $-530-(-557)$. Patel et al. (2014) [41] also used a $70^{\circ}$ endoscope and computed similar MADR values of $-1011 \pm 290$ at a comparable recording rate of $4 \mathrm{kfps}$ (here $-1096 \pm 150$ ). The lower value of $-628 \pm 186$ reported by Bohr et al. (2013) [21] at the same HSV recording rate of $4 \mathrm{kfps}$ may be explained by the lower spatial resolution and that they used a $90^{\circ}$ endoscope. The $90^{\circ}$ endoscope has a larger distance to the glottis than the $70^{\circ}$ endoscope and the glottis area size is naturally smaller in the HSE images. This, in combination with the reduced pixel resolution, reduces the absolute number of pixels in the glottis and hence the pixel changes and therefore the absolute MADR values.

The Open Quotient (OQ) remains constant at 0.97-0.96 for all recording frame rates and is therefore independent of it (group 1). A previous study reported similar values $(0.95 \pm 0.09)[21]$ whereas another study reported slightly lower values $(0.86 \pm 0.17)[41]$.

Significantly lower values were found in other studies: namely, $0.68 \pm 0.12$ [42], $0.64 \pm 0.10$ [33], and $0.57 \pm 0.18$ [43]. Ahmad et al. (2012) [7] also computed lower OQ values (0.68-0.78), which may be explained by the fact that they defined the glottis as fully closed when the opening area of the glottis was less than 5\% of the maximum opening area. Since a posterior glottal chink is common in females, Inwald et al. (2011) [23] found that $47.9 \%$ of healthy females exhibited a small posterior glottal chink and Ahmad et al. may have overestimated the actual "fully closed" time for females.

Similarly, Ikuma et al. (2014) [44] defined the glottis as being closed when the actual glottis area was below $1 \%$ of 

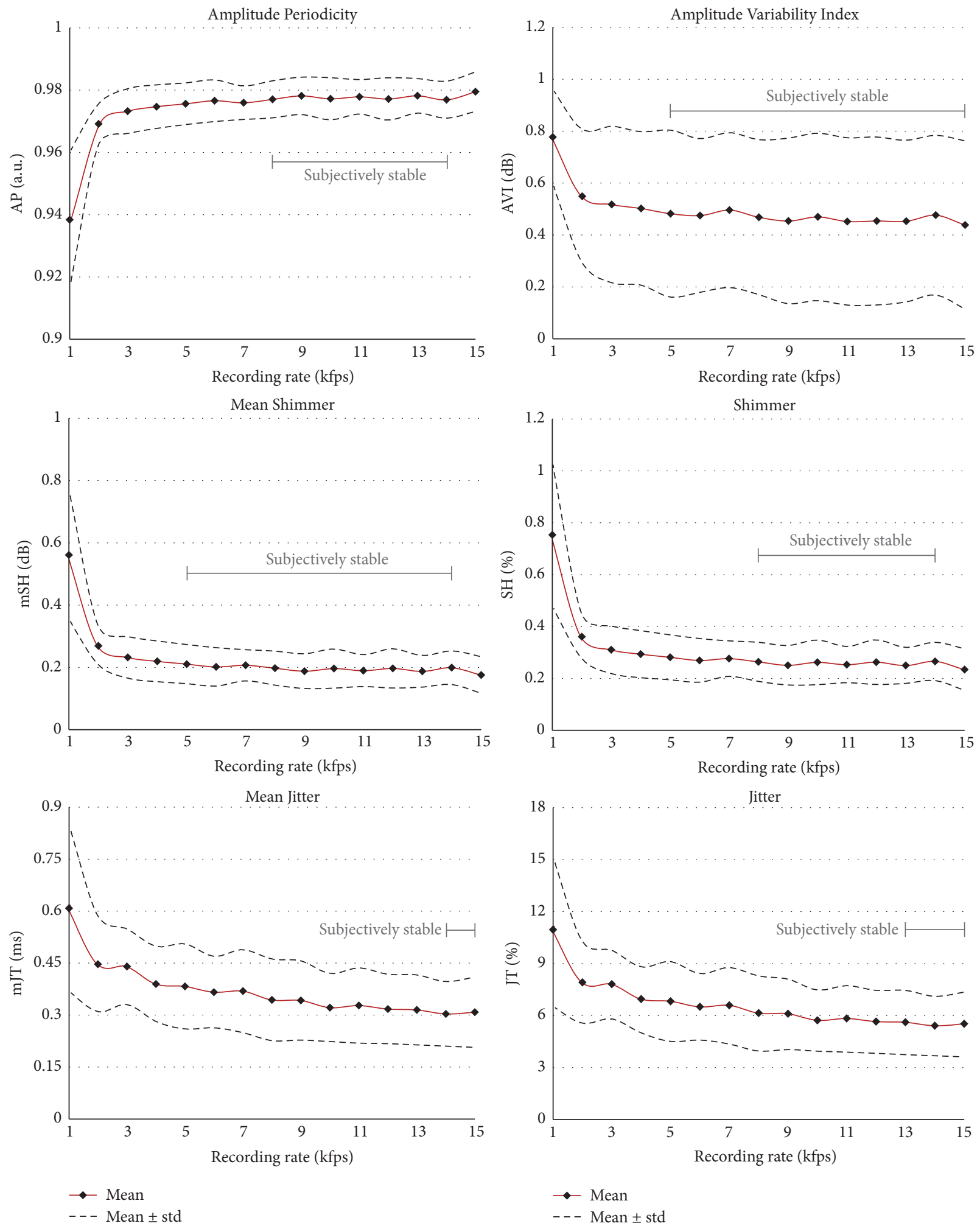

FIGURE 6: The six parameters representing glottal perturbation. Means and standard deviations are given. The subjective rating of stable values over HSV recording frame rates is indicated. 

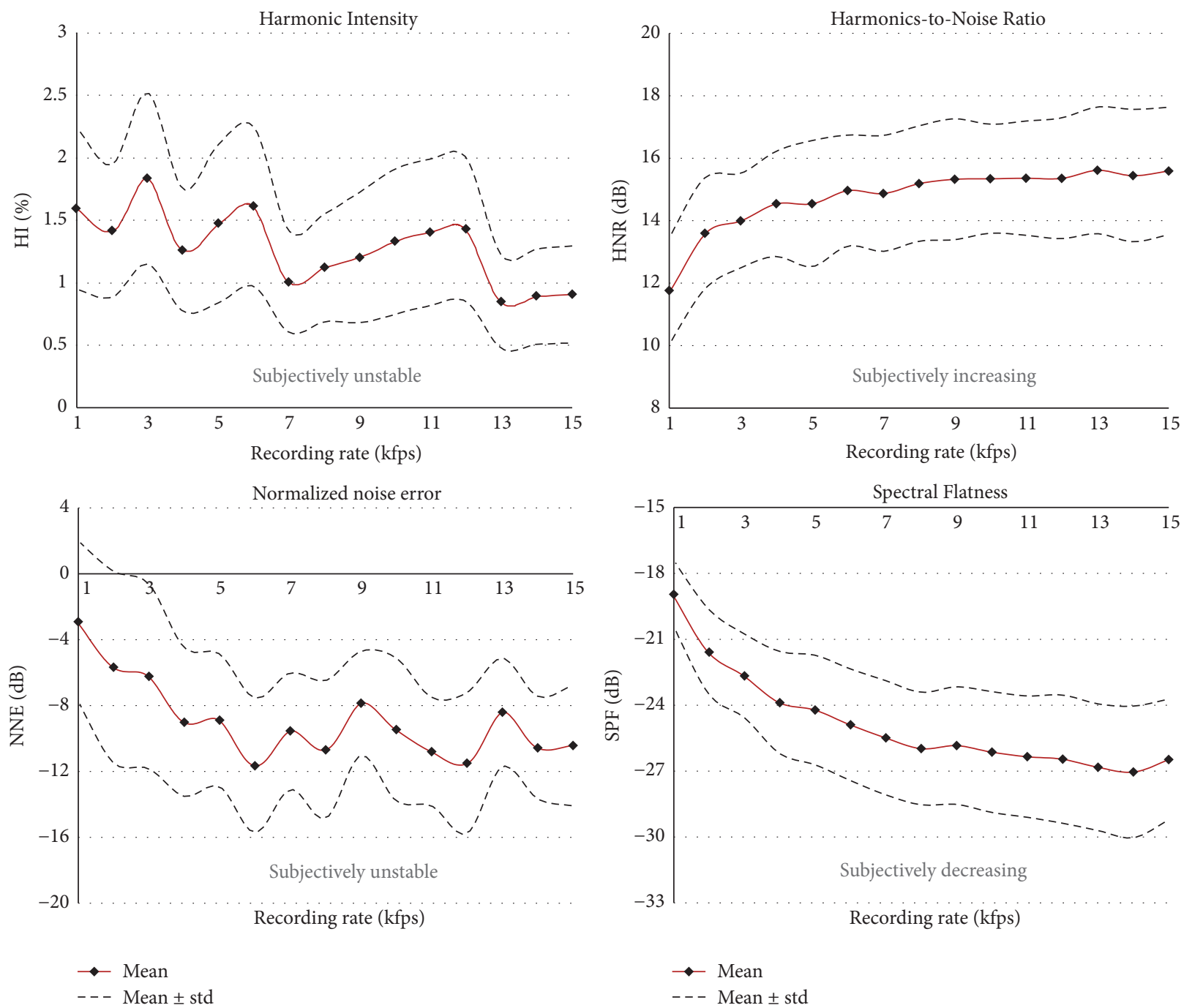

FIGURE 7: Parameters representing glottal harmonic and noise components. The parameters HI, NNE, and SPF are computed on the spectral space of the GAW. Means and standard deviations are given. The obvious, subjectively and objectively determined, instability, and the dependence on the HSV recording rates suggest that HI, NNE, and SPF are not suitable for evaluating the GAW signal.

the maximum glottal area. They computed the OQ for a postsurgery female and male group as $0.91 \pm 0.11$, that is, closer to our values.

We conclude that for OQ it should be defined whether such thresholds will be used and at what cut-off level. Further, depending on the image processing applied, algorithms might consider certain pixels as glottis or not at the state of a nearly closed glottis, which in consequence yields different results for $\mathrm{OQ}$.

For males [6] and children [20], this issue seems to be less important as the posterior glottal chink occurs less commonly, resulting in lower OQ values, Table 5. However, large variations have been reported for OQ. Holmberg et al. (1988) [32] computed the ranges $0.56-0.95$ for normal females and $0.46-0.77$ for normal males using the inverse filtered airflow signal. Hence, it may be difficult to give common valid normative values that might differentiate normal from disordered voices.

The Phase Asymmetry (PA) seems to be equal for all recording frame rates considered (group 1). The computed means in our study vary between -0.02 and -0.01 , reflecting high dynamic left-right symmetry. Zero corresponds to perfect left-right symmetry; that is, the left and right GAW, separated by the glottis axis, reach their maximal area at exactly the same time. Bohr et al. (2013) [21] computed similar values at $-0.02 \pm 0.07$ (they called this parameter "Glottis Phase").

The Rate Quotient (RQ) does not change significantly from $8 \mathrm{kfps}$ (group 2). There, the means are between 1.33 and 1.32. In contrast, Patel et al. (2014) [41] computed much higher values in the range $2.04 \pm 0.78$. These values are also significantly higher than comparable RQ values at $4 \mathrm{kfps}$ in our study (1.41-1.37). One explanation might be the higher phonation fundamental frequency of the subjects in Patel et al.s study compared with ours.

In summary, the RQ values for the typical clinical recording rate $(4 \mathrm{kfps})$ and the highest recording rate differ by only $6 \%$, showing a fairly stable RQ despite statistically significant differences. 


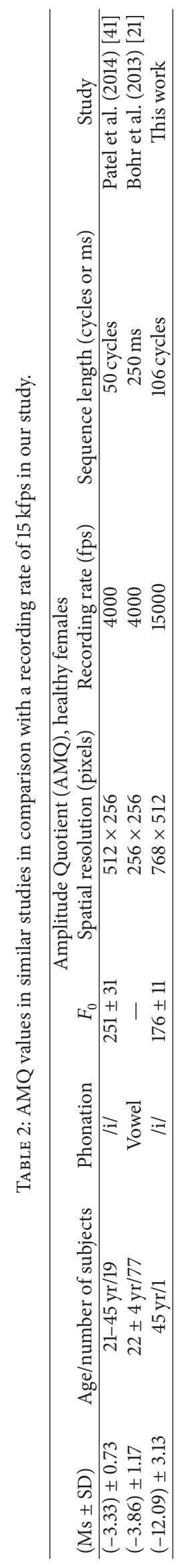




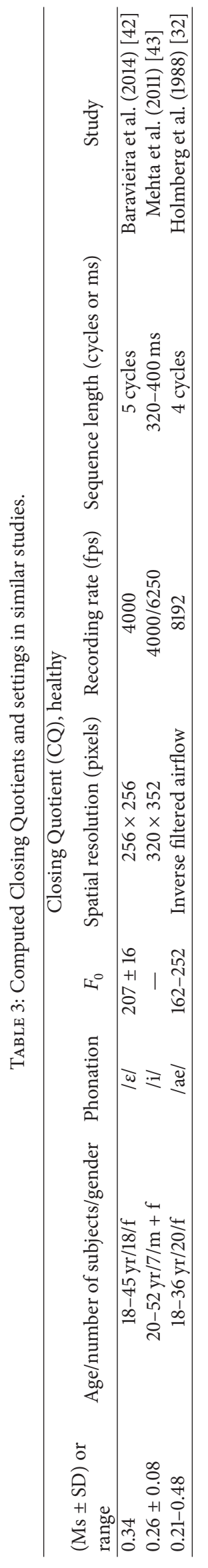




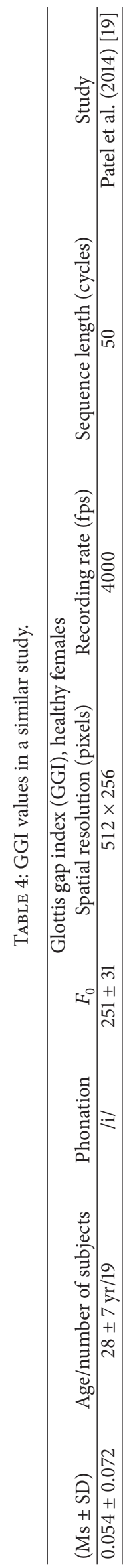




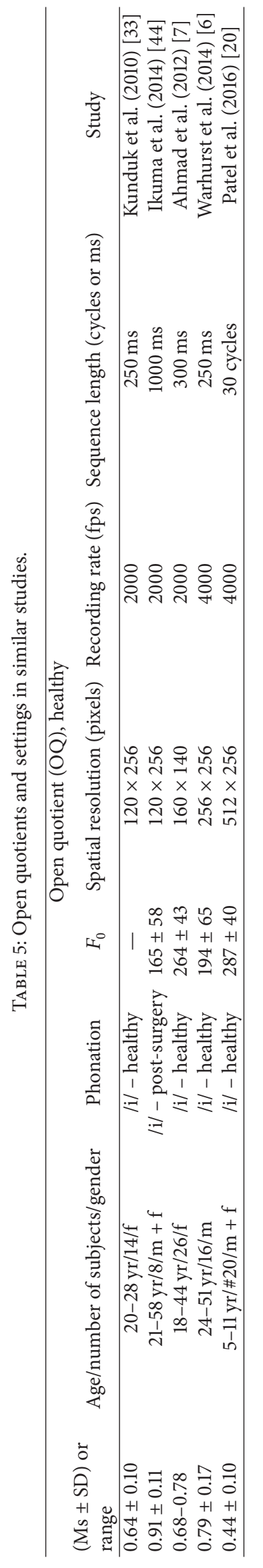


The Speed Index (SI) does not change significantly from $8 \mathrm{kfps}$ and remains at a level of 0.09 (group 2). Ikuma et al. (2014) [44] reported similar values for their cohort (i.e., postsurgery status of a mixed female and male group of eight subjects) at $0.10 \pm 0.07$ for a recording frame rate of $2 \mathrm{kfps}$. The equivalent value in our study at $2 \mathrm{kfps}$ is similar at 0.14 . A higher value of 0.18 was reported elsewhere [41]. Again, this might be explained by the increased mean phonatory fundamental frequency in their subjects. They reported a mean of $251 \mathrm{~Hz}$ [41], which is much higher than in our study $(176 \mathrm{~Hz})$.

The values for SI in our study show fairly large differences with increase in recording rate (i.e., $22 \%$ between 4 and $8 \mathrm{kfps}$ ). Hence, we suggest that a frame rate of $8 \mathrm{kfps}$ and higher should be used to compute SI.

The Speed Quotient (SQ) does not show significant differences from $8 \mathrm{kfps}$ (1.24-1.23). Also, the difference from $4 \mathrm{kfps}(1.28)$ at $4 \%$ is small. In particular, the values of Bohr et al. (2013) [21], with a mean of $1.17 \pm 0.33$, are very similar, although they analyzed a group of young women (age around 20 years). Mehta et al. (2011) [43] also reported similar results for SQ $(1.15 \pm 0.52)$ whereas Kunduk et al. (2010) [33] computed slightly lower values of $0.99 \pm 0.13$. Apart from the statistical analysis, it can be stated that SQ hardly changes between 3 and $15 \mathrm{kfps}$. However, below $3 \mathrm{kfps}$, SQ is significantly higher. Hence, we suggest that, for computing SQ, a recording rate of at least $4 \mathrm{kfps}$ should be used. Interestingly, Holmberg et al. (1988) [32], for the inverse filtered airflow signal, reported much higher values in the range 1.19-2.33. However, these higher values may be explained by inertance effects of the sub- and supraglottal air columns and their interaction during opening and closing of the glottis.

(B) Glottal Periodicity and Perturbation Characteristics. The Amplitude Periodicity (AP) does not show changes between 8 and $14 \mathrm{kfps}$ (group 3). In summary, only the value for a recording rate of $1 \mathrm{kfps}(0.94)$ is obviously lower than the others (0.97-0.98). Despite the statistical differences for the recording rates, it can be assumed that a $2 \mathrm{kfps}$ recording rate might be sufficient. Our values are confirmed by previous work in which AP values of $0.977 \pm 0.009$ were reported [19].

The Amplitude Variability Index (AVI) does not change statistically between 5 and $14 \mathrm{kfps}$ (group 3). Interestingly, at $15 \mathrm{kfps}$ statistically significant differences occur. The mean values for recording rates of 2-4 kfps are slightly higher but basically in a similar range.

The Mean Shimmer $(\mathrm{mSH})$ does not change statistically between 8 and $14 \mathrm{kfps}$ (group 3). Between 2 and $15 \mathrm{kfps}$ the values are fairly similar $(0.27-0.18)$. Only for $1 \mathrm{kfps}$ is the $\mathrm{mSH}$ value greatly increased (0.56). Bohr et al. (2013) [21] computed values of $0.19 \pm 0.08$, which are in the same range as in our study for 3-15 kfps.

For Shimmer (SH), as for $\mathrm{mSH}$, the values do not change statistically between 8 and $14 \mathrm{kfps}$ (group 3). The value at $15 \mathrm{kfps}$ is again statistically different. However, the values change only slightly from $3 \mathrm{kfps}(0.31-0.23 \%)$, being in the same range as those reported by Bohr et al. (2013) [21] of $0.29 \pm 0.13 \%$. However, other studies reported higher values.
Yan et al. (2005) [45] computed 2.1\% for $2 \mathrm{kfps}$, although they did not give the gender or age of the subjects, Table 6 . Ahmad et al. (2012) [7] computed for young healthy females values of $1.5-7.2 \%$ at $2 \mathrm{kfps}$. For older healthy women a high value of $6.2 \%$ was also reported [24]. The above three studies all used a low HSV recording rate of $2 \mathrm{kfps}$, which might be a reason for their different and high values. Also, the factors spatial resolution and number of considered oscillation cycles might have an influence on $\mathrm{SH}$.

The Jitter (JT) values do not change from $10 \mathrm{kfps}(5.8-$ $5.4 \%$ ) (group 2). However, the values for lower recording rates $(10.8-7.8 \%)$ are almost twice as high. The value of $5.7 \pm 3.0 \%$ reported by Bohr et al. (2013) [21] is in the range of values computed in our study for $10 \mathrm{kfps}$ and higher. The comparable value at $4 \mathrm{kfps}$ at $6.9 \%$ is in the range of Bohr et al.

Ahmad et al. (2012) reported lower JT values for older (3.8\%) and younger $(0.9-3.6 \%)$ females [7, 24]. Yan et al. (2005) [45] also computed a lower value of $1.4 \%$. Similarly to $\mathrm{SH}$, further influencing factors seem to be present in computing JT.

The Mean Jitter $(m J T)$ does not change significantly for $10-15 \mathrm{kfps}$ (0.33-0.30 ms) (group 2). The decrease of $51 \%$ over frame rates from 1 to $15 \mathrm{kfps}$ indicates a strong dependence. However, from $4 \mathrm{kfps}(0.39 \mathrm{~ms}) \mathrm{mJT}$ seems to stabilize. Bohr et al. (2013) [21] reported for $4 \mathrm{kfps}$ a mJT of $0.2 \mathrm{~ms}$, being $33 \%$ lower than the value computed here at $15 \mathrm{kfps}(0.30 \mathrm{~ms})$. The difference (49\%) from the corresponding value in our study at $4 \mathrm{kfps}(0.39 \mathrm{~ms})$ is even higher. As for SH and JT, it may not only be the HSV recording rate that influences the computation of $\mathrm{mJT}$.

(C) Harmonics: Noise Signal Components. The Harmonic Intensity (HI), as found statistically, changes over the entire range of HSV recording rates. Further, as seen in Figure 7, HI does not exhibit a definite trend but seems highly unstable. This unstable behavior suggests that HI is not suitable for GAW analysis and therefore should not be considered in future work. No studies of HI applied to the GAW signal were found in the literature.

For the Harmonics-to-Noise Ratio (HNR), statistical analysis showed two stable intervals: 5-9 and 8-15 kfps (group 2). A previous study computed HNR values of $14.9 \pm 5.9 \mathrm{~dB}$ for $4 \mathrm{kfps}$ [21], covering the HNR values over all our recording rates. Significantly higher values were reported by Ikuma et al. (2014) [44] for postsurgical subjects $(37.6 \pm 2.6 \mathrm{~dB})$ and also for healthy subjects $(>32.05 \mathrm{~dB})$ [47]. However, they used a low HSV recording rate $(2 \mathrm{kfps})$ and did not provide age or gender details. The HNR values in the latter two studies are also much higher than those computed for women from the acoustic signal $(24.32 \pm 4.25 \mathrm{~dB})$ [48]. Owing to the higher recording rate of acoustic signals $(44 \mathrm{kHz})$, one would actually expect better HNR values for the acoustic signal. Potentially, the deviation from the earlier studies $[44,47]$ is based on a different HNR computation formula.

For the Normalized Noise Energy (NNE), statistically the values are significantly different for almost all recording rates (group 4), as also confirmed by visual inspection, Figure 7. Between the lowest and highest recording rates there is a difference of $366 \%$, indicating a very high dependence on 


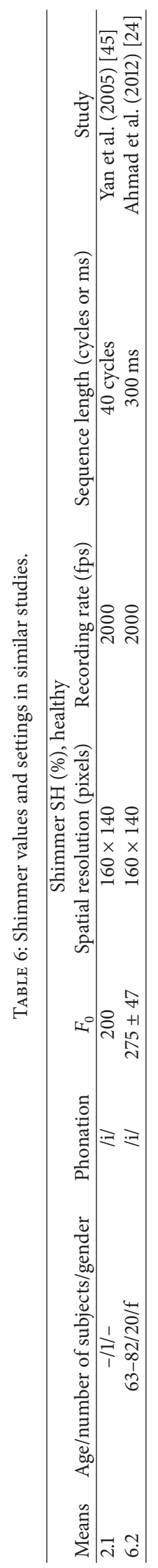


the recording rate. Bohr et al. (2013) [21] computed at $4 \mathrm{kfps}$ a mean NNE of $-10.4 \pm 7.2 \mathrm{~dB}$, similar to the values at 14 and $15 \mathrm{kfps}$ in our study. However, the standard deviation in their study is large and includes NNE values between -3.2 and $-17.6 \mathrm{~dB}$. This interval coincides with the values computed in this study between 1 and $15 \mathrm{kfps}$. These extreme variations indicate that NNE values computed on the GAW signal might not be clinically applicable.

The Spectral Flatness (SPF) is found to be statistically and subjectively unstable (group 4), Figure 7. In summary, the values change from -18.9 to -26.5 , representing a difference of $29 \%$. This unstable behavior, as already seen for the three previous parameters computed on the spectral space, does not favor clinical applications in the future.

\section{Recommendation for HSV Recording Rates}

Taking a closer look at the typical recording frame rate of $4 \mathrm{kfps}$ used in clinical HSV settings, it can be seen that all parameters, except OQ and PA (group 1), change at higher recording rates, Figure 1 . These findings concur with considerations by Patel et al. (2016) [20] and it raises the question of whether for these parameters the computed values at $4 \mathrm{kfps}$ reflect the actual vibratory vocal fold characteristics or whether they are only a more or less accurate approximation of the real vocal fold dynamics. However, the relative small parameter changes between 4 and $15 \mathrm{kfps}$ for the parameters in $(A)$ glottal dynamic characteristics (except AMQ and MADR) and (B) glottal periodicity and perturbation characteristics (Figures 4-6) justify the application of a recording rate of $4 \mathrm{kfps}$ in clinical studies and daily clinical routine.

Nevertheless, it should be stated that, from a recording rate of $8 \mathrm{kfps}, 11$ parameters (55\%) do not change statistically or show only negligible differences at higher recording rates. Hence, in scientific studies where the exact vocal fold characteristics are of interest, we recommend a recording rate of at least $8 \mathrm{kfps}$, and higher if possible. In other words, based on the fundamental frequencies of the subject in this study being close to $200 \mathrm{~Hz}$, the results suggest that the frame rate of the camera should be 40 times higher than the vocal fold oscillations to achieve stable values for certain dynamic parameters.

\section{Limitations}

Several limitations are evident in this study. The recordings for only one subject were analyzed. However, for each frame rate from 1000 to $15000 \mathrm{fps}$ for 20 initial recordings resulting in a total of $300 \mathrm{HSV}$ data sets were analyzed to support the findings of this study. Every effort was made to ensure similarity in the endoscope-vocal fold distance, and the effects of varying endoscope-vocal fold distance on the parameters investigated here need to be considered in a further study. In addition, the HSV recordings were carried out in one session; hence, it is not known if fatigue factors were influencing the variability of HSV parameter values.
The recordings of only one subject were analyzed, which allows the conclusion that parameters are dependent on the HSV recording rate if they show statistically significant differences for different recording rates, but this does not enable us to conclude definitely and universally that the parameters that did not show differences will do so in a large cohort. Also, the interval boundaries where dependences on the recording rate were found and the corresponding parameter values (Figure 1) cannot be generalized based on one subject. Hence, an extended study, considering age and gender, will have to be performed to confirm the observed behavior with respect to different HSV recording frame rates.

Further, the influence of the spatial HSV resolution will have to be investigated in the future. The various studies discussed here exhibit a wide range of spatial HSV resolution that might also be responsible for the different computed parameter values.

The number of oscillation cycles considered might also play a role in HSV parameter variability, as seen before in acoustic signals [49]; for example, the studies discussed here reported between around 4 and 110 oscillation cycles.

The influence of the applied segmentation techniques on the GAW and hence on the absolute parameter values is of interest and will have to be analyzed in the future. Depending on the HSV image quality, different image processing algorithms may yield slightly different segmentation results and hence GAW curves. This directly transfers to the computed GAW parameters. In our study, we eliminated this error source by eliminating HSV movies with low image quality.

\section{Conclusion}

It was shown that the majority (90\%) of the investigated parameters are dependent on the HSV recording rate. The influence of the recording rate on HSV parameters varied differently and clearly. Hence, this is a factor not to be neglected during quantitative HSV analysis. To overcome this issue and to bring HSV into clinical routine, we suggest further investigations of potential influencing factors such as HSV recording rate, HSV spatial resolution, number of considered vocal fold oscillations, and gender and age of the subjects. From there, normative tables, if needed separated for these factors, could be introduced. Finally, this study showed that the commonly used HSV recording rate of $4 \mathrm{kfps}$ may be insufficient to distinguish the vibratory vocal fold characteristics in detail.

\section{Disclosure}

The funder had no role in the study design, data collection and analysis, decision to publish, or preparation of the manuscript.

\section{Competing Interests}

The authors declare that there is no conflict of interests regarding the publication of this paper. 


\section{Acknowledgments}

This study was supported by the Deutsche Krebshilfe (http:// www.krebshilfe.de/nc/startseite.html) under Grant no. 111332.

\section{References}

[1] E. Van Houtte, K. Van Lierde, E. D’Haeseleer, and S. Claeys, "The prevalence of laryngeal pathology in a treatment-seeking population with dysphonia," The Laryngoscope, vol. 120, no. 2, pp. 306-312, 2010.

[2] M. Fukahori, S.-I. Chitose, K. Sato et al., "Regeneration of vocal fold mucosa using tissue-engineered structures with oral mucosal cells," PLoS ONE, vol. 11, no. 1, Article ID e0146151, 2016.

[3] C. R. Krausert, A. E. Olszewski, L. N. Taylor, J. S. McMurray, S. H. Dailey, and J. J. Jiang, "Mucosal wave measurement and visualization techniques," Journal of Voice, vol. 25, no. 4, pp. 395-405, 2011.

[4] D. D. Deliyski, P. P. Petrushev, H. S. Bonilha, T. T. Gerlach, B. Martin-Harris, and R. E. Hillman, "Clinical implementation of laryngeal high-speed videoendoscopy: challenges and evolution," Folia Phoniatrica et Logopaedica, vol. 60, no. 1, pp. 33-44, 2008.

[5] E. M. Yiu, J. Kong, R. Fong, and K. M. Chan, "A preliminary study of a quantitative analysis method for high speed laryngoscopic images," International Journal of Speech-Language Pathology, vol. 12, no. 6, pp. 520-528, 2010.

[6] S. Warhurst, P. McCabe, R. Heard, E. Yiu, G. Wang, and C. Madill, "Quantitative measurement of vocal fold vibration in male radio performers and healthy controls using high-speed videoendoscopy," PLoS ONE, vol. 9, no. 6, Article ID e101128, 2014.

[7] K. Ahmad, Y. Yan, and D. M. Bless, "Vocal fold vibratory characteristics in normal female speakers from high-speed digital imaging," Journal of Voice, vol. 26, no. 2, pp. 239-253, 2012.

[8] C. T. Herbst, J. Unger, H. Herzel, J. G. Švec, and J. Lohscheller, "Phasegram analysis of vocal fold vibration documented with laryngeal high-speed video endoscopy," Journal of Voice, 2016.

[9] H. S. Shaw and D. D. Deliyski, "Mucosal wave: a normophonic study across visualization techniques," Journal of Voice, vol. 22, no. 1, pp. 23-33, 2008.

[10] M. Döllinger, "The next step in voice assessment: high speed digital endoscopy and objective evaluation," Current Bioinformatics, vol. 4, no. 2, pp. 101-111, 2009.

[11] A. Ziethe, R. Patel, M. Kunduk, U. Eysholdt, and S. Graf, "Clinical analysis methods of voice disorders," Current Bioinformatics, vol. 6, no. 3, pp. 270-285, 2011.

[12] T. Shinghal, A. Low, L. Russell, E. J. Propst, A. Eskander, and P. Campisi, "High-speed video or video stroboscopy in adolescents: which sheds more light?" Otolaryngology-Head and Neck Surgery, vol. 151, no. 6, pp. 1041-1045, 2014.

[13] H. S. Bonilha, D. D. Deliyski, J. P. Whiteside, and T. T. Gerlach, "Vocal fold phase asymmetries in patients with voice disorders: a study across visualization techniques," American Journal of Speech-Language Pathology, vol. 21, no. 1, pp. 3-15, 2012.

[14] S. Hertegård and H. Larsson, "A portable high-speed camera system for vocal fold examinations," Journal of Voice, vol. 28, no. 6, pp. 681-687, 2014.
[15] J. Lohscheller, H. Toy, F. Rosanowski, U. Eysholdt, and M. Döllinger, "Clinically evaluated procedure for the reconstruction of vocal fold vibrations from endoscopic digital high-speed videos," Medical Image Analysis, vol. 11, no. 4, pp. 400-413, 2007.

[16] O. Gloger, B. Lehnert, A. Schrade, and H. Völzke, "Fully automated glottis segmentation in endoscopic videos using local color and shape features of glottal regions," IEEE Transactions on Biomedical Engineering, vol. 62, no. 3, pp. 795-806, 2015.

[17] G. Andrade-Miranda, J. I. Godino-Llorente, L. Moro-Velázquez, and J. A. Gómez-García, "An automatic method to detect and track the glottal gap from high speed videoendoscopic images," BioMedical Engineering OnLine, vol. 14, no. 1, article 100, 2015.

[18] A. Yamauchi, H. Imagawa, K.-I. Sakakibara et al., "Characteristics of vocal fold vibrations in vocally healthy subjects: analysis with multi-line kymography," Journal of Speech, Language, and Hearing Research, vol. 57, no. 2, pp. S648-S657, 2014.

[19] R. Patel, D. Dubrovskiy, and M. Döllinger, "Characterizing vibratory kinematics in children and adults with high-speed digital imaging," Journal of Speech, Language, and Hearing Research, vol. 57, no. 2, pp. 674-686, 2014.

[20] R. R. Patel, H. Unnikrishnan, K. D. Donohue, and H. Liu, "Effects of vocal fold nodules on glottal cycle measurements derived from high-speed videoendoscopy in children," PLoS ONE, vol. 11, no. 4, Article ID e0154586, 2016.

[21] C. Bohr, A. Kraeck, U. Eysholdt, A. Ziethe, and M. Döllinger, "Quantitative analysis of organic vocal fold pathologies in females by high-speed endoscopy," The Laryngoscope, vol. 123, no. 7, pp. 1686-1693, 2013.

[22] S. Hertegård, H. Larsson, and T. Wittenberg, "High-speed imaging: applications and development," Logopedics Phoniatrics Vocology, vol. 28, no. 3, pp. 133-139, 2003.

[23] E. C. Inwald, M. Döllinger, M. Schuster, U. Eysholdt, and C. Bohr, "Multiparametric analysis of vocal fold vibrations in healthy and disordered voices in high-speed imaging," Journal of Voice, vol. 25, no. 5, pp. 576-590, 2011.

[24] K. Ahmad, Y. Yan, and D. Bless, "Vocal fold vibratory characteristics of healthy geriatric females-analysis of high-speed digital images," Journal of Voice, vol. 26, no. 6, pp. 751-759, 2012.

[25] S. Petermann, S. Kniesburges, A. Ziethe, A. Schützenberger, and M. Döllinger, "Evaluation of analytical modeling functions for the phonation onset process," Computational and Mathematical Methods in Medicine, vol. 2016, Article ID 8469139, 10 pages, 2016.

[26] M. Echternach, M. Döllinger, J. Sundberg, L. Traser, and B. Richter, "Vocal fold vibrations at high soprano fundamental frequencies," Journal of the Acoustical Society of America, vol. 133, no. 2, pp. EL82-EL87, 2013.

[27] I. Titze, Workshop on Acoustic Voice Analysis: Summary Statement, National Center for Voice and Speech, Iowa City, Iowa, USA, 1995.

[28] M. P. Karnell, K. D. Hall, and K. L. Landahl, "Comparison of fundamental frequency and perturbation measurements among three analysis systems," Journal of Voice, vol. 9, no. 4, pp. 383-393, 1995.

[29] J. Unger, J. Lohscheller, M. Reiter, K. Eder, C. S. Betz, and M. Schuster, "A noninvasive procedure for early-stage discrimination of malignant and precancerous vocal fold lesions based on laryngeal dynamics analysis," Cancer Research, vol. 75, no. 1, pp. 31-39, 2015.

[30] P. Alku, M. Airas, E. Björkner, and J. Sundberg, "An amplitude quotient based method to analyze changes in the shape of the 
glottal pulse in the regulation of vocal intensity," Journal of the Acoustical Society of America, vol. 120, no. 2, pp. 1052-1062, 2006.

[31] N. Henrich, G. Sundin, D. Ambroise, C. d'Alessandro, M. Castellengo, and B. Doval, "Just noticeable differences of open quotient and asymmetry coefficient in singing voice," Journal of Voice, vol. 17, no. 4, pp. 481-494, 2003.

[32] E. B. Holmberg, R. E. Hillman, and J. S. Perkell, "Glottal airflow and transglottal air pressure measurements for male and female speakers in soft, normal, and loud voice," Journal of the Acoustical Society of America, vol. 84, no. 2, pp. 511-529, 1988.

[33] M. Kunduk, M. Döllinger, A. J. McWhorter, and J. Lohscheller, "Assessment of the variability of vocal fold dynamics within and between recordings with high-speed imaging and by phonovibrogram," Laryngoscope, vol. 120, no. 5, pp. 981-987, 2010.

[34] I. R. Titze, "Theoretical analysis of maximum flow declination rate versus maximum area declination rate in phonation," Journal of Speech, Language, and Hearing Research, vol. 49, no. 2, pp. 439-447, 2006.

[35] R. J. Baken and R. F. Orlikoff, Clinical Measurement of Speech and Voice, Cengage Learning, 2nd edition, 1999.

[36] Q. Qiu, H. K. Schutte, L. Gu, and Q. Yu, "An automatic method to quantify the vibration properties of human vocal folds via videokymography," Folia Phoniatrica et Logopaedica, vol. 55, no. 3, pp. 128-136, 2003.

[37] S. Bielamowicz, J. Kreiman, B. R. Gerratt, M. S. Dauer, and G. S. Berke, "Comparison of voice analysis systems for perturbation measurement," Journal of Speech, Language, and Hearing Research, vol. 39, no. 1, pp. 126-134, 1996.

[38] J. Lessing, Entwicklung einer Klassifikationsmethode zur akustischen Analyse fortlaufender Sprache unterschiedlicher Stimmgüte mittels Neuronaler Netze und deren Anwendung [Ph.D. thesis], Georg-August-Universität Göttingen, Mathematisch-Naturwissenschaftlich Fakultät, Göttingen, Germany, 2007.

[39] E. Yumoto, W. J. Gould, and T. Baer, "Harmonics-to-noise ratio as an index of the degree of hoarseness," Journal of the Acoustical Society of America, vol. 71, no. 6, pp. 1544-1550, 1982.

[40] H. Kasuya, S. Ogawa, K. Mashima, and S. Ebihara, "Normalized noise energy as an acoustic measure to evaluate pathologic voice," Journal of the Acoustical Society of America, vol. 80, no. 5, pp. 1329-1334, 1986.

[41] R. R. Patel, D. Dubrovskiy, and M. Döllinger, "Measurement of glottal cycle characteristics between children and adults: physiological variations," Journal of Voice, vol. 28, no. 4, pp. 476486, 2014.

[42] P. B. Baravieira, A. G. Brasolotto, A. Hachiya, M. T. TakahashiRamos, D. H. Tsuji, and A. N. Montagnoli, "Comparative analysis of vocal fold vibration using high-speed videoendoscopy and digital kymography," Journal of Voice, vol. 28, no. 5, pp. 603-607, 2014.

[43] D. D. Mehta, M. Zañartu, T. F. Quatieri, D. D. Deliyski, and R. E. Hillman, "Investigating acoustic correlates of human vocal fold vibratory phase asymmetry through modeling and laryngeal high-speed videoendoscopy," Journal of the Acoustical Society of America, vol. 130, no. 6, pp. 3999-4009, 2011.

[44] T. Ikuma, M. Kunduk, and A. J. McWhorter, "Objective quantification of pre- and postphonosurgery vocal fold vibratory characteristics using high-speed videoendoscopy and a harmonic waveform model," Journal of Speech, Language, and Hearing Research, vol. 57, no. 3, pp. 743-757, 2014.
[45] Y. Yan, K. Ahmad, M. Kunduk, and D. Bless, "Analysis of vocal-fold vibrations from high-speed laryngeal images using a Hilbert transform-based methodology," Journal of Voice, vol. 19, no. 2, pp. 161-175, 2005.

[46] C. Bohr, A. Kräck, D. Dubrovskiy et al., "Spatiotemporal analysis of high-speed videolaryngoscopic imaging of organic pathologies in males," Journal of Speech, Language, and Hearing Research, vol. 57, no. 4, pp. 1148-1161, 2014.

[47] T. Ikuma, M. Kunduk, and A. J. McWhorter, "Advanced waveform decomposition for high-speed videoendoscopy analysis," Journal of Voice, vol. 27, no. 3, pp. 369-375, 2013.

[48] M. Döllinger, M. Kunduk, M. Kaltenbacher et al., "Analysis of vocal fold function from acoustic data simultaneously recorded with high-speed endoscopy," Journal of Voice, vol. 26, no. 6, pp. 726-733, 2012.

[49] J. Hohm, M. Döllinger, C. Bohr, S. Kniesburges, and A. Ziethe, "Influence of $F_{0}$ and sequence length of audio and electroglottographic signals on perturbation measures for voice assessment," Journal of Voice, vol. 29, no. 4, pp. 517.e11-517.e21, 2015. 


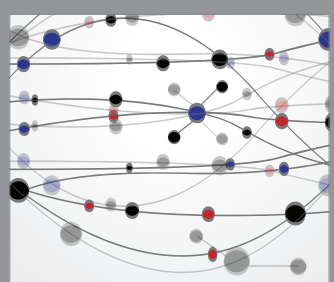

The Scientific World Journal
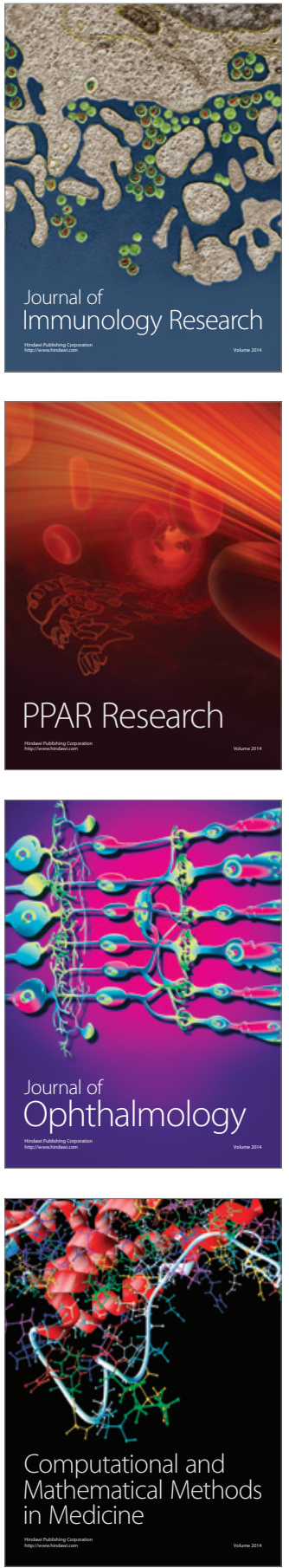

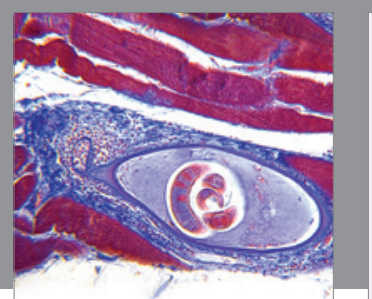

Gastroenterology Research and Practice

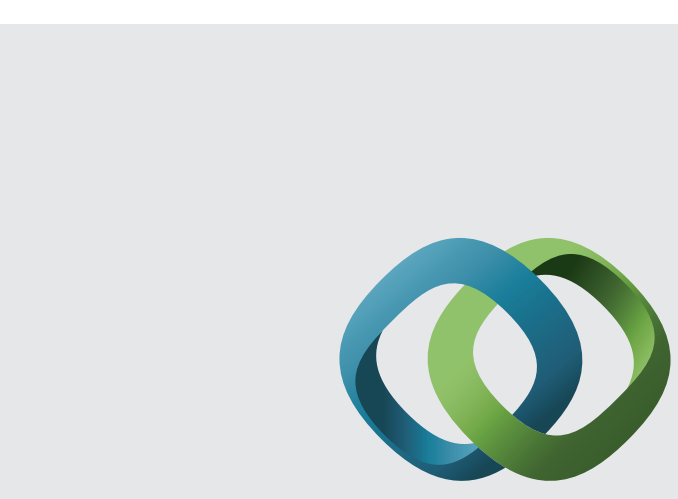

\section{Hindawi}

Submit your manuscripts at

http://www.hindawi.com
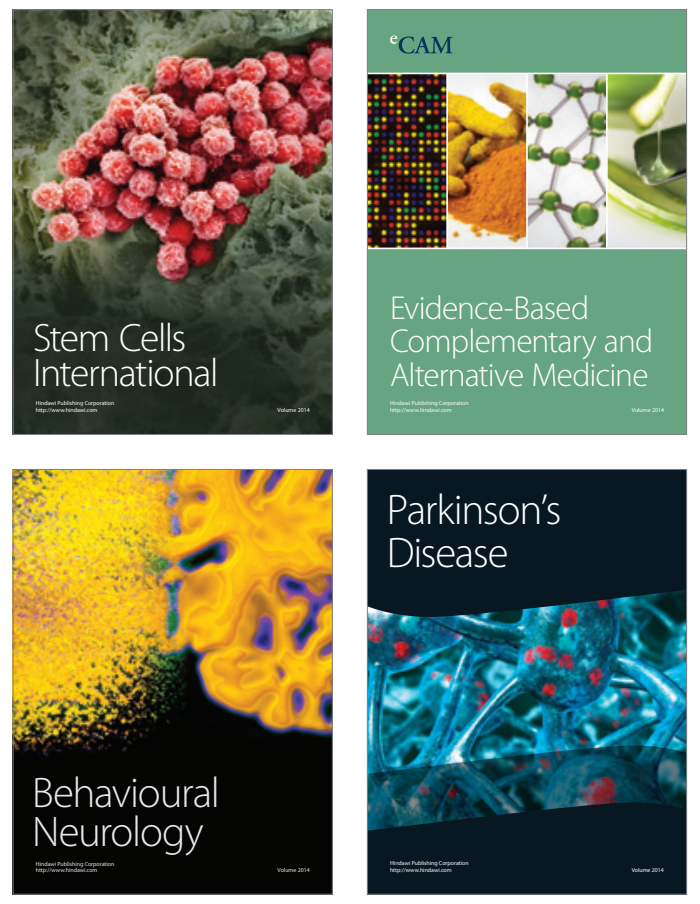
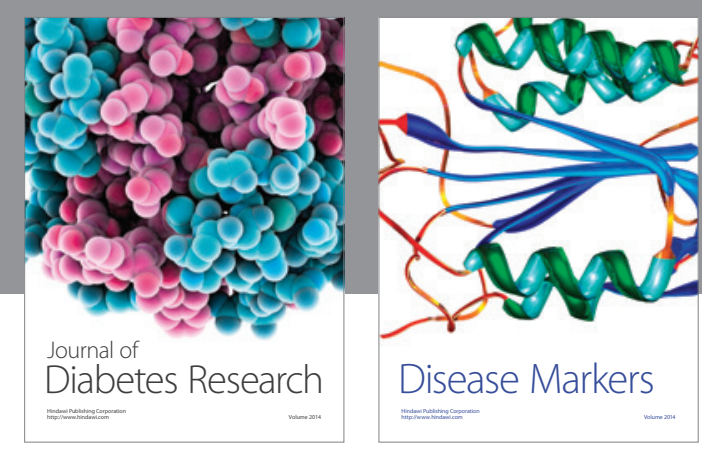

Disease Markers
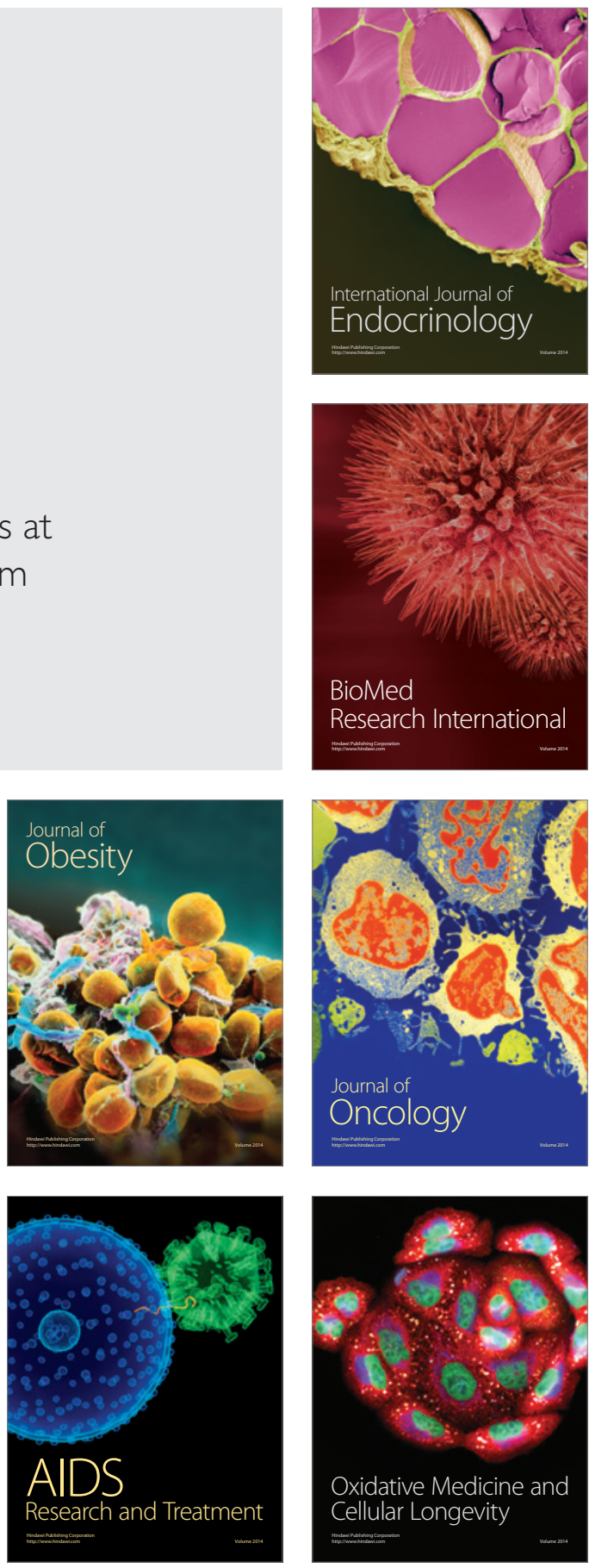